\title{
Sexualidad y Educación Superior en Brasil y Cuba coordenadas analíticas para un estudio comparativo
}

\section{Cláudia Ramos de Souza Bonfím \\ Professora da Faculdade Dom Bosco (Cornélio Procópio/PR) \\ Eduardo Francisco Freire Roach \\ Professor da Universidad Agraria de La Habana (Cuba)}

\section{Resumen}

Como se puede observar, desde el siglo XVIII hasta el comienzo de la década de los 60 , la construcción social de la sexualidad y la educación sexual en Brasil y Cuba, transcurren por similares circunstancias socioeconómicas, políticos y culturales, asociadas a los regimenes feudales y capitalistas hegemónicos coloniales, neocoloniales, republicanos, pseudorepublicanos, y dictatoriales. Sin embargo, posterior a los 60 se evidencia rupturas entre los sistemas de educación superior de ambos países. Brasil pasa de una dictadura militar al neoliberalismo, y Cuba de la dictadura al socialismo. No obstante, tanto en los momentos de continuidad como en los de ruptura, los educadores han encarados los mismos problemas ancestrales de la sexualidad y similares enfoques de educación sexual, que van desde los tradicionalistas, hasta los biologistas, humanistas y participativos.

Palabras-clave: Sexo; Sexualidad; Educación Sexual.

\begin{abstract}
As can be seen, from the eighteenth century to the beginning of the ' 60 s, the social construction of sexuality and sex education in Brazil and Cuba, carried by similar socio-economic, political and cultural regimes associated with the feudal and capitalist hegemonic colonial, neocolonial, Republicans, pseudo republicans and dictatorial. However, after 60 shows the breakdown between higher education systems of both countries. Brazil goes a military dictatorship to new Liberalism, and Cuba from dictatorship to socialism. However, in the moments of continuity in the burst, educators have faced the same problems ancestral sexuality and similar approaches to sex education, ranging from traditional to organic, humanistic and participatory.
\end{abstract}

Keywords: Gender; Sexuality; Sex Education. 


\section{Introducción}

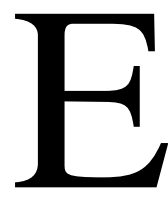
1 sexo y la educación sexual han sido temas recurrentes en la historia de la humanidad. Puede decirse las culturas y civilizaciones se han caracterizado por el modo en que encaran el sexo, ya sea desde el punto de vista de las relaciones sexuales o de las representaciones que de las mismas se hacen los actores sociales: los individuos hombres y mujeres adolescentes, adultos y ancianos, así como los grupos, colectivos, comunidades, las instituciones, el Estado, la sociedad civil, la escuela, etc.

A primera vista la finalidad biológica del sexo oscila entre la reproducción y el deseo o placer. Pero, como hombres y mujeres somos seres esencialmente sociales, el sexo es en esencia una construcción social, y en tal sentido hablamos de sexualidad. El término de sexo podría continuar designando un acto biológico que realizan los seres vivos en general, incluyendo a los seres humanos. Por lo tanto, sexualidad no es igual a sexo. El sexo entre seres humanos es en esencia sexualidad.

De antaño y no menos hoy en día, la problemática de la anticoncepción, el aborto, la dinámica reproductiva, así como las enfermedades de transmisión sexual (ETS), suscitan reflexión y debate sobre la sexualidad y la educación sexual. Por eso ha tenido relevancia la concepción medico higienista de la sexualidad, que a fin de cuenta reduce la sexualidad al sexo. Pero con Nunes (1996 y 2006), apostamos a que hay que ir más allá, transitando hacia un enfoque de la sexualidad humana como construcción histórica, política y cultural, que manifiesta potencialidades como expresión plena de la identidad humana, y sus valores de respeto, libertad, igualdad, justicia y solidaridad.

Tal desafío implica también la consideración de las múltiples y complejas dimensiones de la sexualidad: desde las eróticas, subjetivas, reproductivas, hasta en lo que tiene que ver con sus significaciones y consecuencias, la responsabilidad social y la autonomía propia y ajena. Se trata, en fin, de la comprensión de la sexualidad en la trama compleja de la cultura y las relaciones sociales. 
Tributando a este enfoque de la sexualidad como totalidad o como fenómeno complejo exige un marco analítico filosófico y científico social, que de cuenta de cómo la sexualidad y la educación sexual se construyen en correspondencia con determinadas circunstancias históricas asociadas a las estructuras socioeconómicas y sistemas políticos. Esta relación explica el modo en que se encara la educación sexual en las sociedades.

Existen diferencias en el modo de representación de la sexualidad entre las sociedades. En no menor medida estas se manifiestan al interior de las sociedades, ya sea en su evolución histórica, o en los diversos y múltiples marcos en que transcurre la vida social.

Uno de esos marcos es el sistema educacional, y dentro de este el sistema de educación superior donde a través de los profesores y los futuros profesores se construyen y se vehiculan imágenes (hegemónicas y contra hegemónicas) de la sexualidad hacia los niveles anteriores. El intercambio de experiencias y los estudios comparados ofrecen un marco idóneo para tratar este asunto en toda su complejidad.

Los autores de este trabajo que estamos enfrentando dicho desafío científico, provenimos de realidades socioeconómicas, políticas y culturales diferentes: Brasil y Cuba, dos países que se diferencian en cuando a estructura y dinámica socioeconómica, política y cultural, así también en cuanto a sistemas de educación en general y superior en particular.

En esta ponencia hemos querido articular nuestras reflexiones acerca de la imagen de la sexualidad y de la educación sexual que se construye en nuestras instituciones de educación superior, fundamentalmente en nuestras universidades, y en el contexto de la preparación de profesores que enseñan Biología. De ahí que la interrogante fundamental de este estudio sea la siguiente: ¿Qué semejanzas y diferencias existen entre la educación superior en Brasil y en Cuba en cuanto al modo de construir y vehicular la sexualidad?

Partimos de la imagen de la sexualidad como construcción social sugerida en los pensamientos de autores como Freud, Reich, Marcuse, Foucault y Nunes. 
Como muy bien se conoce, Freud en su teoría y practica del psicoanálisis colocó énfasis en la sexualidad infantil, la cual no limitó a la función de los órganos genitales, sugiriendo, además la intervención de una serie de actividades que proporcionan placer mas allá de la satisfacción fisiológica.

Reich (1897-1957), que parte de Freud, pero asumiendo a Marx, profundiza en la relación sexo/sociedad y sexo/ideología, puntualizando que el sexo es un factor de control social. Para Reich el hombre es la única especie donde el sexo no se rige absolutamente por las leyes biológicas. Esto lo lleva a la elaboración de toda una crítica radical de la vida sexual de la humanidad en los marcos del mundo burgués y capitalista.

Marcuse, por su parte, advierte que la represión sexual es una de las características más importantes de las sociedades donde prima la explotación del hombre por el hombre. De ahí que hablara de la miseria no solo económica, sino también sexual propia de esas sociedades. Así también se refería a cierto embotellamiento de la sensualidad como atrofia y embrutecimiento de todos los órganos del ser humano. A fin de cuentas, para Marcuse, la represión sexual contribuye a la manutención de un orden social represivo desde todos los puntos de vistas. Según él, modo de reducir la agresividad y la destructividad generada por la represión es hacer que no sea sublimizada, es decir, que se permita una sexualidad libre, más intensa y polimorfita.

Foucault (1997) establece una interesante conexión entre la sexualidad y los juegos de saber-poder, mediante los cuales esta interviene en el control de los cuerpos, en función a su vez del aparato de producción, de forma tal que se garantice el ajuste de la población a los procesos económicos. Para Foucault la sexualidad articulada a los saberes y poderes fue un factor importante en la consolidación del modo capitalista de producción, en tanto funciona como una especie de biopoder, por el cual se hacen inversiones políticas proyectadas hacia el control conceptual y practico de la sexualidad. Esto responde según Foucault a la socialización del cuerpo como fuerza de trabajo, que conduce a entenderlo como una realidad biopolítica. La medicina, el urbanismo, la demografía, y también la escuela, resultan estrategias biopolíticas. 
De los autores reseñados se desprende que la sexualidad y la educación sexual involucran factores socioeconómicos, políticos y culturales, cuya manifestación y entendimiento son marcados precisamente por las transformaciones ocurridas en el modo de producción de la sociedad, envolviendo dimensiones no solo objetivas y biológicas, sino también subjetivas y sociales.

Concordamos con Nunes (1996) y Nunes y Silva (2000) que la sexualidad en la escuela requiere de una concepción científica y humanística e histórico-cultural. Este enfoque requiere considerar la escuela como una de las instituciones que conforman al aparato ideológico del Estado, mediante el cual se cautela la reproducción de los valores sociales, y donde se instalan mecanismos y dispositivos de control de nuestras conductas y representaciones.

Por lo tanto, la educación en general y la educación sexual en particular de forma conceptual o utópica, se manifiesta como un factor de desenvolvimiento humano y social, pero también como agente masificante y excluyente como acontecen en muchos contextos sociales.

Es con este enfoque que hemos encarado la discusión sobre la sexualidad y la educación sexual en el contexto de la educación superior en nuestros países.

\section{Sexualidad y Educación Sexual en Brasil}

La educación superior en Portugal y Brasil existe desde hace dos siglos. Las primeras escuelas superiores datan de 1760. Desde la fundación de la Universidad de Coimbra en Portugal, hasta la Reforma Universitaria Pombalina se mantiene un modelo educativo contrarreforma, cuya función era la formación de teólogos, sacerdotes, juristas e administradores del imperio portugués, dentro de la orientación estricta de la Orden de los Jesuitas y la Iglesia Católica. Lógicamente, la construcción y vehiculación de la sexualidad y la educación sexual se encuentran marcadas en el siglo XVIII por los discursos ideológicos, religiosos, filosóficos, científicos, médicos, 
jurídicos, artísticos, literarios, y pedagógicos, articulados a las concepciones católicas y jesuitas institucionalizadas.

Considerando a Foucault (1997), tenemos que entonces la población constituía un problema económico político, por lo que la preocupación relativa a la sexualidad adviene como necesidad de control de la tasa de natalidad, la edad del casamiento, la legitimidad de la descendencia, la precocidad y frecuencia de las relaciones sexuales, así el celibato y las practicas contraceptivas. La clase hegemónica entendería que la manutención de tal condición, de sus propiedades y de su fortuna, están vinculadas al modo en que cada cual vive sus relaciones sexuales. De entonces que la educación sexual se presenta como un principio regulador de la población y de intervención y control de la sexualidad.

La historia de Brasil ilustra muy bien estas articulaciones de la sexualidad con los factores sociales. Por ejemplo, la forma de concubinato que predominada en América Latina entre las clases rurales y criollas, fue atajada por la Iglesia católica, que como sabemos defendía la familia patriarcal, y solo toleraba (no aceptaba) el sexo por placer a los hombres y mujeres marginales (Werebe, 1984, apud Goldberg, 1984).

Una constante en estos tiempos seria el establecimiento de diferencias entre hombres y mujeres en torno a como encarar la vida sexual. Obviamente, la educación sexual construirá y vehiculará una imagen de la sexualidad femenina y masculina que contribuirá a la preasignación de papeles y a las brechas de géneros, que favorecerían la posición de liderazgo del padre en la familia (Vanconcellos, 1915 apud Bruschini e Barroso, 1982).

Ya para 1915, comienzan a llegar al Brasil desde Europa influencias de las corrientes medico-higienistas, que introducían la idea de la educación sexual como un factor para el combate de la masturbación y las enfermedades venéreas (hoy denominadas enfermedades de transmisión sexual o ETS).

Si bien, el régimen colonial prácticamente silenciaba los discursos sobre el sexo que no le eran afines, con la Republica, nace en el XIX, según Foucault, una especie de nueva tecnología del sexo que se distancia de la 
ideología eclesiástica, y que se estructura a partir de tres ejes: la medicina, la demografía y la pedagogía. En la Republica se comienza a hablar más abiertamente sobre el sexo, pero es para garantizar el control de la natalidad, el crecimiento de la población, y las ETS.

En esta época proliferan los discursos que defienden una educación sexual orientada hacia la planificación familiar, la maternidad libre, y hasta inclusive se dan corrientes que llevan la preocupación por la degeneración de la raza humana (Werebe, 1998).

En década de los 30, durante el gobierno de Getúlio Vargas, con la reforma de Francisco Campos (1932), se establece que la educación superior puede ser administrada por instituciones no solo oficiales, sino también particulares patrocinadas o no por los gobiernos federal y estatales (Sampaio, apud Silva, 2001). Pero la educación sexual era destinada solo a los "muchachos de valor", excluyéndose a las muchachas, las cuales deberían mantenerse inocentes e iniciadas sexualmente, y destinada a la conformación de una buena familia. A las prostitutas se les ve como una tentación a los muchachos (Chauí, 1984).

En opinión de Guimarães (1995), en las décadas de los 40 y los 50 nada se hizo en materia de educación sexual, dada la severa represión de la Iglesia Católica. No se tenía acceso libre a los libros médicos sobre sexualidad, y en las casas, por lo general, estas obras eran ocultadas a los jóvenes.

Sin embargo, a inicios de los 60 soplaron vientos favorables para la educación sexual en Brasil (Guimarães, 1995). En diversos entornos educativos, sobre todo superiores, se buscaba atender a las reivindicaciones del alumnado, sobre todo en lo que respecta a las explicaciones biológicas de la sexualidad. La política fatalista oficial propugnaba entonces que les fuera vedado a los profesores de Biología y Sociología que defendieran la limitación de la concepción. La educación sexual se relegaba a aspectos de reproducción, maternidad y desenvolvimiento corporal, es decir se enfatizaba en los aspectos biológicos de la sexualidad (Goldberg, 1984).

Es bien sabido que después del 1964 se instaura en Brasil una educación que responde al régimen de capitalismo dependiente, donde el modelo educativo es elaborado por especialistas norte-americanos, y en función del 
capital, y la reproducción de las clases sociales, la fuerza de trabajo y mano de obra que requiere, (Freitag, 1979). Pero este no sin ser contradictorio periodo democrático (1946-1964) fue interrumpido por un violento golpe militar desencadenado contra el gobierno de João Goulart.

Aun en ese tiempo continuaba la influencia de la política natalista que como vimos coloca el énfasis en la cuestión de la reproducción, la natalidad, el desenvolvimiento corporal, en fin, que atendía los aspectos biológicos de la sexualidad (Bruns y Santos, 2000).

En 1968 se hace una propuesta de introducción de la educación sexual en todas las escuelas de nivel primario y secundario. Esta propuesta no fructificó dado el clima de represión social que existía en las escuelas. Los profesores que se arriesgaban a tocar temas de sexualidad eran perseguidos. Se argumentaba que la inocencia sexual era la mejor defensa de la pureza y la castidad.

Con los 70 irrumpe un clima de mayor censura de los proyectos de educación sexual, pero estos comienzan a parecer en la segunda mitad de esta década gracias a los movimientos feministas, los movimientos de control de la población, y también dadas las mudanzas en el comportamiento juvenil. Entonces comienzan a parecer en las escuelas contenidos de fisiología y anatomía de la sexual, del ciclo menstrual, la fecundación, la gestación, y de las enfermedades venéreas.

Es precisamente en las universidades donde se gusta todo un movimiento de resistencia contra la dictadura militar. Pero la Reforma universitaria de 1968, seguía siendo la prolongación del sistema político dictatorial militar (Gadotti, 1980), y se hacia acompañar de los procesos de modernización y de cierto mimetismo cultural, que escondían una estructura conservadora (Wanderley, 1999), de índole tecnocratica.

Con el derrumbe de la dictadura militar, y entre 1983 a 1985 se hacen públicos casos de SIDA. La muerte por esta enfermedad de personalidades como Lauro Corona, Cazuza, entre otros, provoca preocupaciones e interés para la divulgación de medios preventivos. Ante esta avasalladora realidad material e histórica, la educación sexual fue asimilada en la educación y en la sociedad como una cuestión curativa. Digamos que ese interés 
no nació de una propuesta emancipatoria de valoración de las potencialidades corporales, por lo mantenía y ganaba espacio una visión medico higienista y biologista de la sexualidad (Nunes, 1996).

Para la década de los 90 tenemos una universidad brasilera operacional o universidad "Mc-Donald's" influenciada por el neoliberalismo (Chauí, 2001, p. 190). En estas circunstancias se banaliza y se mercantiliza la sexualidad en función del modo de producción capitalista, de la reproducción de los papeles sexuales dominantes (Nunes, 1996).

Entonces vemos como la educación sexual se liga preferentemente a las cuestiones de salud pública, el control de la natalidad, la regulación demográfica, y la reproducción de un ser humano como fuerza de trabajo y mano de obra.

Irrumpe así una especie de educación sexual meramente descriptiva y biologista (Nunes, 1987). En estas condiciones los medios enfatizan en la realización de los deseos sexuales, no resaltando lo relativo a la responsabilidad y a las consecuencias de la vida sexual. Predomina aun la tensión entre religión, familia, y libertad sexual. Y los discursos positivistas y naturalista sobre la sexualidad acaparan la dinámica ideológica de las escuelas.

Mucho énfasis se hace en el tema de la constitución anatómica y funcional de los genitales, lo cual a nuestro modo de ver viene muy a tono con el boom de la "estética pornográfica" ampliamente aceptada en la práctica en los países capitalistas.

Por su fuera poco, aun perviven elementos de representación de la sexualidad como pecado, que hace que muchas personas vivencien la sexualidad no de un modo placentero, y mas bien solo como reproducción de la especie.

Tiene lugar con mucha fuerza la educación de patrones duales de comportamiento sexual según los géneros. Por ejemplo, se vehicula mucho la visión de que la sexualidad masculina debe ser desde el inicio más cuantitativa en cuanto a besos y contactos sexuales, a diferencia de que la sexualidad de las muchachas. Esta construcción de la sexualidad obedece a una visión patriarcal y machistas de las identidades sexuales, donde la mujer 
debe ver el sexo como un acto de amor, en función del matrimonio y de la familia.

Pues bien, toda este apretado recorrido por la historia de la educación sexual en Brasil, muestra como dadas las improntas socioeconómicas por la que ha atravesado la sociedad brasilera (colonia, republica, dictadura, neoliberalismo), la sexualidad ha estado atrapada entre la tradición y la modernización. En ambos casos queda el camino zanjado para una comprensión social y cultural de la sexualidad que supere las visiones conservadoras, medico-higienista, biologistas, mecanicistas, mercantilistas, tecnocráticas, y machistas de la sexualidad.

\section{Sexualidad y Educación Sexual en Cuba}

Ahora vamos a ser un recorrido mas apretado aun de la historia de la educación sexual en el contexto de la educación superior en Cuba, enfatizando en como el sistema social educativo socialista que Cuba vehicula desde inicios de los 60, vehicula una imagen de la sexualidad, que apunta a la superación de las visiones tradicionalistas, biologistas, mecanicistas, mercantilistas, tecnocráticas, y machistas de la sexualidad.

Con el triunfo sin precedentes de la izquierda guerrillera insurgente y donde el estudiantado universitario jugo un papel fundamental, en 1959 aparecen las primeras iniciativas de introducción de la educación sexual en los sistemas de enseñanza (Mederos, 1994 y Leyva, 1994). Antes de entonces los gobiernos coloniales, neocoloniales, pseudorepublicanos y dictatoriales silenciaba todo discurso sobre la sexualidad que no estuviera enmarcado en tabúes tradicionalistas o enfoques medico-higienistas y curativos de la sexualidad. Sin temor a equivocarnos podemos decir que las representaciones sociales que predominaban de la sexualidad antes del 59, se asemejaban a las que imperaba en la historia de la educación sexual en Brasil que hemos descritos en el epígrafe anterior.

Un antecedente importante de cómo hoy en día se encara la sexualidad y la educación sexual en Cuba es la creación en 1959 del Grupo Nacional de Trabajo de Educación Sexual (GNTES), que surgió con el objetivo no 
solo de informar, sino también de formar a las nuevas generaciones en una concepción de la sexualidad que va mas allá de su función reproductiva estrechamente enmarcada en la pubertad. Desde entonces ha sido una constante la intención que se les brinde a niños y niñas, y a los hombres y mujeres adultos y ancianos, una información sexual acorde a su edad, de forma parte que en todo momento no se inhiba, y más bien se enriquezca su esfera psicosexual. La política educacional del estado socialista ha estado consciente de no descuidar la educación sexual y que esta debe trascender el placer sexual individual, y el conocimiento sobre la anatomía y fisiología genital (Álvarez, 1998).

Este Grupo sentó importantes pautas ideológicas, epistemológicas e metodológicas para que las organizaciones que la auspiciaron: la Federación de Mujeres Cubanas (FMC), así como los Ministerios de Salud y Educación, y otras organizaciones juveniles, políticas y de masas, encaran el desafió de la educación sexual.

Con la institucionalización de la educación sexual en todos los niveles de enseñanza, se multiplicaron las iniciativas para canalizar este tipo de educación, como la publicación de libros y la importación de cursos sobre sexualidad.

La educación sexual en Cuba tiene gran alcance dada la relevancia que se le ha dado a la educación como factor del cambio social hacia una sociedad socialista y soberana. El ideal educativo martiano y marxista que matizaba la eolítica educacional, conlleva a una educación sexual muy conectada con la preparación del ser humano para la vida, y para enseñarle a pensar. La educación se enmarca dentro de toda una estrategia educativa en favor de promover el desarrollo integral y autorrealización de la personalidad.

Es recurrente en el discurso político cubano actual sobre la sexualidad el que esta se contemple dentro de los principios de la nodiscriminación, libertad, responsabilidad (González, 1997), comprensión, tolerancia, reciprocidad, participación, respeto a la individualidad (Mederos, 1994 y Leyva, 1994). Pero es importante destacar que esta proyección educativa no se reduce al tratamiento academicista de la sexualidad. 
La iniciativa del GNTS, le siguió su transformación en 1989 en Centro Nacional de Educación Sexual (CENESEX), cuyo encargo o misión social consiste en coordinar e implementar el desarrollo del Programa $\mathrm{Cu}-$ bano de Educación Sexual, que de una forma u de otra pauta las iniciativas de educación sexual a través todos los niveles, y que se relaciona con otros programas que también tributan a la educación sexual: El Programa de la Maternidad y la Paternidad Responsable, el Programa de Planificación Familiar, el Programa de Prevención de Infecciones de Transmisión Sexual y SIDA, y el Programa de Consultas de Orientación y Terapia Sexual, así como el Programa de Prevención y Erradicación del Consumo de Drogas ilícitas y el Alcoholismo.

En Cuba, dado el carácter gratuito y de amplio acceso de la educación superior y también de la atención a la salud, y los medios de información y comunicación, se viabiliza el alcance que puede tener una educación sexual no reduccionista y emancipadora.

No obstante, lejos estamos de decir que en Cuba todo lo relativo a la sexualidad y la educación sexual es color de rosas. Hoy en desafió la educación sexual encara problemas asociados a la sexualidad que se creían habían sido erradicados con la Revolución y el proceso socialista. Por ejemplo, este es el caso de la problemática que gira en torno a la anticoncepción y la interrupción del embarazo (el aborto).

Como se conoce que en 1985, la Revolución despenalizó el aborto, y creo garantías hospitalarias excepcionales para prevenir y evitar sus riesgos. Sin embargo, a pesar de esta medida se hizo acompañar de los programas de educación sexual, y contribuyo a la disminución de muerte materna por aborto indebido, se hizo uso de esta intervención de una forma indiscriminada. Las tasas cubanas de aborto son altas (Gonzalez et al, 2001), y no faltaron hombres y mujeres que lo identificaban con un mero método anticoncepcional de primera mano, y preferibles al uso del condón, las pastillas anticonceptivas o los dispositivos intrauterinos. Se estima que más de 70 por ciento de las cubanas que acuden a una consulta de infertilidad, para lograr un embarazo tienen como antecedente uno o más abortos durante la adolescencia o en la etapa de adulta joven (Acosta, 2006). 
En la década de los 90 se registro un descenso de los abortos, a causa de múltiples factores el aumento del uso de la regulación menstrual. Lógicamente, la educación sexual generalizada jugo un papel importante en este descenso.

Esta problemática del aborto se articula en parte con la de disminución del tiempo de duración de los matrimonios y los divorcios. En Cuba el matrimonio y su disolución son un derecho de hombres y mujeres, y resulta un proceder jurídico poco costoso. El tiempo promedio de duración del matrimonio en Cuba viene siendo alrededor de 10 a 14 anos. Se ha registrado que por cada 100 matrimonios se tiene una tasa de divorcio de 69,9\%. Desde el año 2000 la tasa de divorcio fluctúa entre 3 y 3,4 por cada mil habitantes, y no existe tendencia al aumento; el índice es alto, pero estable», aseguró Enrique González, director del Centro de Estudios de Población y Desarrollo de la Oficina Nacional de Estadísticas. Estudios realizados en Cuba sobre este problema muestran que en este fenómeno esta incidiendo no solo las facilidades de realizar la separación, sino también a la inadecuada selección de la pareja. Otro elemento importante es el casamiento entre adolescentes.

Otro problema que esta desafiando a la educación sexual en todos los niveles de enseñanza, es el del aumento entre adolescente de las ETS, dado el alto porcentaje de no-uso del condón, los hábitos oro-genitales y genito anal (Cortez, 1999), así como el aumento del cambio frecuente de parejas. Este ha sido un problema que existe en Cuba desde la época prerrevolucionaria, y que estuvo relacionado a la prostitución (Farinas et al, 2001). Estudios que se hicieron en los 70 mostraron como en la Cuba revolucionaria la tendencia de enfermedades ETS como la sífilis fue ascendente en los últimos 35 anos. En el caso del VIH o SIDA, la tasa de prevalencia se estima oficialmente en un $0,05 \%$, y ha sido reconocida como una de las bajas del mundo. El programa de atención de esta pandemia reporta que 3969 cubanas viven con $\mathrm{VIH}$, donde el $54 \%$ recibe atención ambulatoria.

En Cuba se discute intensamente el tema de la prostitución que había sido superado con su prohibición tras el triunfo de la Revolución, pero que se mantuvo, y que mostró caras no antes vistas a partir de la situación eco- 
nómica en que se encontró Cuba a finales de los 80 y principios de 10590 (Elizalde, 2003). Tanto las familias como el vecindario evitan usar el termino estigmatizante de prostitución. Se tiende hablar de "jineteras" o de "jineteros".

Por ultimo, no queríamos pasar por alto el auge que hoy en día se muestra en el mundo y en Cuba por el tema de la bisexualidad y la homosexualidad. En 1970 se abolió la homosexualidad como figura delictiva. Pero este ha sido un tema tabú, inclusive durante la Revolución, en que si bien no se institucionalizo la discriminación, tampoco se hizo mucho por erradicar los prejuicios estigmatizando contra los homosexuales.

Hoy en día como nunca antes están emergiendo abiertamente discursos contra tales prejuicios. Se observan condiciones favorables para la aceptación de los homosexuales, y a su derecho a manifestar su preferencia sexual libremente. Hoy la educación sexual esta enrumbada a que esta tolerancia se convierte en respeto a las preferencias sexuales de las personas, y a la integración de estas (no la segregación) con la población heterosexual en igualdad de condiciones (Jimenez, 2003).

La educación sexual en las universidades se ha convertido en un tema que atraviesa las funciones sustantivas (docencia, investigación y extensión). Atendiendo los acuciantes problemas que giran en torno a la sexualidad: la alta tasa de abortos en jóvenes, la alta tasa de matrimonios, divorcios y madres solteras, las relaciones prematrimoniales promiscuas, la extrema precocidad de la primera relación sexual y el incremento de enfermedades de transmisión sexual, entre otras.

En las universidades sexuales cubanas como tendencia existe una cátedra, grupo de o centro de investigación que realizan actividades de rectoras de Educación sexual, para la familia, para la equidad de género, al interior y extramuros de las universidades, tanto en las pedagógicas (Castellanos, 2000) como en las no pedagógicas.

A través de la historia de las universidades cubanas han desfilado diferentes enfoques de cómo encarar la educación sexual. Podríamos, siguiendo a Castro (2001) reseñar cuatro enfoques. El tradicional o de adoctrinamiento moral, autoritario y verticalista (de los 60 a los 70); el enfoque pre- 
ventivo o conductista, experimentalista pedagógico y medicalista (de los 80 a los 90), orientado hacia la habitación del embarazo no deseado, y de las ETS; el enfoque humanista, que destaca el reconocimiento de la equidad sexual, la aceptación de las diferencias individuales, la consideración a los grupos minoritarios más vulnerables (de los 90 al 2000); y el enfoque participativo que pretende destacar el carácter activo de los sujetos de la Educación Sexual (a partir del 2000). Se vincula con las corrientes sobre la Educación Popular desarrolladas en el continente. Este enfoque declara dirigir las acciones de educación sexual para satisfacer necesidades básicas de aprendizaje.

Los institutos superiores pedagógicos, es decir, las universidades pedagógicas, se rigen por el Proyecto Cubano "Educación formal para un comportamiento sexual responsable", que auspicia el Ministerio de Educación (MINED) cuyo enfoque es enfoque humanista crítico, alternativo y participativo, definido inicialmente. Similar enfoque se aplica también en el caso de las universidades no pedagógicas, por ejemplo, la de la Habana y la de Villa Clara, donde existen centros de referencia nacional en materia de educación sexual.

\section{Conclusiones}

Como se puede observar, desde el siglo XVIII hasta el comienzo de la década de los 60, Brasil y Cuba, son dos países que transcurren por similares circunstancias socioeconómicas, políticos y culturales, asociadas a los regimenes feudales y capitalistas hegemónicos coloniales, neocoloniales, republicanos, pseudorepublicanos, y dictatoriales.

Resultan también similares la ocurrencia de problemas que giran en torno a la sexualidad, tales como el crecimiento poblacional, la familia, las brechas de géneros, la prostitución, las enfermedades ETS, las violaciones sexuales, el embarazo no deseado, el aborto, la precocidad de las relaciones sexuales, el embarazo la problemática de los prejuicios ante la diversidad de preferencias sexuales, etc. 
Tanto en Brasil como en Cuba han tenido lugar enfoques de la educación sexual que van desde los tradicionalistas inspirados en la doctrina oficial de la Iglesia católica, como los inspirados en la modernización que tienden a un enfoque biologicista de la sexualidad y cientificista tecnocrático, medicalista-higienista y conductista de la educación sexual.

A partir de los 60 se evidencias rupturas entre las realidades socioeconómicas y políticas entre Brasil y Cuba. Brasil queda sumergido en un régimen dictatorial de corte militarista que conllevó a una educación sexual sesgada por el silencio discursivo impuesto represivamente, y orientado tanto en un sentido tradicionalista como en un sentido medicalista. Con el derrumbe de la dictadura soplaron vientos democráticos liberales y neoliberales, que si bien significaron una apertura de la educación sexual, instauraron una imagen de la sexualidad mercantilista con énfasis en el placer sexual genital.

En Cuba tenemos a partir de esa década un proceso que fue transitando hacia el socialismo, abriendo así posibilidades para una visión integral, no reduccionista, y emancipadora de la sexualidad y la educación sexual. La Revolución creó instituciones y programas que atendían la problemática de la sexualidad, ya sea desde el punto de vista medico, como social. No obstante, en la Cuba revolucionaria se asumieron enfoques mas tradicionalistas y medicalistas, hasta enfoques más humanistas y participativos.

A pesar de las desemejanzas históricas, la educación superior de ambos países encara los problemas ancestrales de la sexualidad, y proyecta la búsqueda de enfoques más efectivos y eficaces de educación sexual, atendiendo a las realidades particulares de su labor profesional.

\section{Referencias Bibliográficas}

ÁLVAREZ LAJONCHERE, C. Educación sexual en Cuba. Sex Soc 4(6) 1998;78.

BRUNS, Maria Alves de Toledo; SANTOS, Claudiene. A educação sexual pede espaço. São Paulo: Ômega, 2000. 
BRUSCHINI C.; BARROSO, C. Educação sexual: debate aberto. Petrópolis: Vozes, 1982.

CASTELlANOS ONATE, Carlos Manuel, VERA NODA, Carmen Rosa, GUTIERREZ VENEGAS, Pedro et al. Educación sexual en un grupo de estudiantes secundarios. Rev Cubana Med Gen Integr. [online]. ene.-feb. 2000, vol.16, no.1 [citado 06 Octubre 2007], p.31-38. Disponible en la World Wide Web: $<$ http://scielo.sld.cu/scielo.php?script=sci_arttext\&pid=S086421252000000100006\&lng=es\&nrm=iso >. ISSN 0864-2125.

CASTRO ALEGRET PEDRO LUIS. Familia y educación sexual. Enfoques investigativos.Revista Cubana de Psicología. Vol.21, No.2, 2004

CHAUÍ. Marilena. Escritos sobre a universidade. São Paulo: UNESP, 2001.

. Repressão sexual: essa nossa (des)conhecida. 6 ed. São Paulo: Brasiliense, 1984.

CORTES ALFARO, Alba, GARCIA ROCHE, René G., HERNANDEZ SANCHEZ, Mariela et al. Encuesta sobre comportamientos sexuales riesgosos y enfermedades de transmisión sexual en estudiantes adolescentes de Ciudad de La Habana, 1996. Rev Cubana Med Trop. [online]. mayo-ago. 1999, vol.51, no.2 [citado 05 Octubre 2007], p.120-124. Disponible en la World Wide Web: $<$ http:/ / scielo.sld.cu/scielo.php?script=sci_arttext\&pid=S0375-

07601999000200010\&lng=es\&nrm=iso >. ISSN 0375-0760.

ELIZALDE ROSA MIRIAM. ¿Crimen o castigo? La Jiribilla, http://www.lajiribilla.cu/2003/n122 09/122 09.html. 2003.

FOUCAULT, Michel. A vontade de saber. Rio de Janeiro: Jorge Zahar, 1997.

FREITAG, Bárbara. Escola, Estado e Sociedade. 3 ed. SP: Cortez \& Moraes, 1979. GADOTTI, Moacir. Educação e poder. São Paulo: Editora Moraes, 1980.

GARCÍA JIMÉNEZ EDUARDO. La sociedad cubana ante la homosexualidad. Alma Mater (http://www.almamater.cu/ ) 2003.

GOLDBERG, M. A. A. Educação Sexual: uma proposta - um desafio. São Paulo: Cortez, 1984.

GONZALEZ LABRADOR, Ignacio y MIYAR PIEIGA, Emilia. La Educación Sexual en la Enseñanza Médica Superior. Rev Cubana Med Gen Integr. [online]. Mayo-jun. 2002, vol.18, no.3 [citado 05 Octubre 2007], p.223-225. Disponible en la World Wide Web: 
$<$ http://scielo.sld.cu/scielo.php?script=sci_arttext\&pid=S0864-

21252002000300011\&lng=es\&nrm=iso >. ISSN 0864-2125.

GONZÁLEZ HA, CASTELLANOS SB. En: Hacia una sexualidad responsable y feliz. Documento teórico metodológico. Ciudad Habana: Ed. Pueblo y Educación; 1997:10-.

GUIMARÃES, I. Educação Sexual na Escola: mito e realidade. Campinas: Mercado de Letras, 1995.

LEYVA Y. La educación sexual en Cuba antes y después de 1959. En: Algunos temas sobre sexualidad. La Habana: Editorial Ciencias Médicas, 1994:12-7.

MEDEROS M. La educación en población desde la educación de la sexualidad en la escuela cubana. Sexol Soc 1994;1(0):16-7.

MEDEROS, M. Educación sexual. Sex Soc 4(2):1998; 20-1.

NUNES, César Aparecido. Desvendando a Sexualidade. Campinas, SP: Papirus, 1987.

- Filosofia, Sexualidade e Educação: as relações entre os pressupostos ético-sociais e bistórico-culturais presentes nas abordagens institucionais sobre a educação sexual escolar (Tese de Doutorado) - Faculdade de Educação, UNICAMP, 1996.

.; SILVA, Edna. A Educação Sexual da Criança. Campinas, São Paulo: Autores Associados, 2000.

SILVA, Alberto Carvalho da. Alguns problemas do nosso ensino superior. Revista Estudos Avançados, ed. Especial, v 15, no 42, EAP, maio/agosto 2001, p. $269 / 291$.

WANDERLEY, Luiz Eduardo W. O que é Universidade. São Paulo: Brasiliense, 1999. Coleção Primeiros Passos.

WEREBE, Maria José Garcia. Sexualidade, Política, Educação. Campinas, SP: Autores Associados, 1998. 